\title{
A barrier to diffusion
}

Stimulation of excitatory neurons triggers the movement of calcium into dendritic spines and the build up of second messengers in the spine head, which, in turn, activates regulatory signalling cascades that underpin alterations in the strength of excitatory synapses. The level of signalling molecules in the spine head is therefore crucial for the induction of synaptic plasticity, and Bloodgood and Sabatini have now shown that the spine neck has a dynamic role as a diffusion barrier in regulating the movement of these molecules into and out of the spine head.

These researchers combined twophoton laser-scanning microscopy with two-photon laser photoactivation to measure regulation of diffusion of the photoactivatable green fluorophore PAGFP, which is similar in size to many proteins that are involved in synaptic modification, across spine necks in individual pyramidal neurons of the rat hippocampus. They showed that there are substantial variations in the time taken for diffusion across the spine neck among different spines, whereas within individual spines there was a high degree of consistency in these values.
In a subset of spines, there was very little movement across the spine neck, indicating that this structure can act as a barrier to diffusion, effectively isolating the spines from the dendrites. Repeated measurements over long time periods showed that this effect is reversible, which indicates that the diffusion barrier imposed by spine necks is dynamic and not static.

Bloodgood and Sabatini proposed that the variations in rate of diffusion across spine necks depend on differences in neuronal and synaptic activity. As changes in activity influence the number of AMPA ( $\alpha$-amino-3-hydroxy-5-methyl-4isoxazole propionic acid) receptors at the synapse, they assessed the effects of receptor blockade on rates of diffusion. Suppressing activation by blocking AMPA receptors led to rapid escape of proteins from the spine head into the dendritic shaft, which, they speculate, decreases the likelihood of plasticity induction. By contrast, increasing activation by blocking $\mathrm{GABA}_{\mathrm{A}}$ ( $\gamma$-aminobutyric acid type $A$ ) receptors hindered diffusion across the spine neck, thereby maintaining important signalling molecules in the spine head and increasing the likelihood of plasticity induction. This latter effect was not attributable to inhibition of $\mathrm{GABA}_{\mathrm{A}}$ receptors alone, but also seemed to depend on secondary changes in the rate of firing or on glutamate transmission.

Only the cross-sectional area of the neck, and not the volume of the spine head, the length of the neck or the diffusion coefficient of the molecules, accounted for the effects of activity on diffusion rates. Moreover, in experiments that involved the green-fluorescing pyranine-based fluorophore HPTS, which is similar in size to second messengers such as cyclic AMP, diffusion across the spine neck was further restricted by pairing synaptic stimulation with back-propagating action potentials.

These findings support an important and dynamic role for the spine neck in the regulation of movement of second messengers and activated proteins into and out of the spine head, and therefore in the induction of synaptic plasticity. It is hoped that future work will reveal the factors that directly influence constriction or expansion of spine necks.

Alison Rowan

ORIGINAL RESEARCH PAPER Bloodgood, B. L. $\&$ Sabatini, B. L. Neuronal activity regulates diffusion across the neck of dendritic spines. Science 310, 866-869 (2005) FURTHER READING Segal, M. Dendritic spines and long-term plasticity. Nature Rev. Neurosci. 6 , 277-284 (2005) 